\title{
On the implementation and effectiveness of morphological close-open and open-close filters for topology optimization
}

\author{
Mattias Schevenels • Ole Sigmund
}

Received: date / Accepted: date

\begin{abstract}
This note reconsiders the morphological closeopen and open-close filters for topology optimization introduced in an earlier paper (Sigmund, 2007). Close-open and open-close filters are defined as the sequential application of four dilation or erosion filters. In the original paper, these filters were proposed in order to provide length scale control in both the solid and the void phase. However, it was concluded that the filters were not useful in practice due to the computational cost of the sensitivity analysis. In this note, it is shown that the computational cost is much lower if the sensitivity analysis for each erosion or dilation step is performed sequentially. Unfortunately, it is also found that the close-open and open-close filters do not have the expected effect in terms of length scale control: each close or open operation ruins the effect of the preceding filters, resulting in a design with a minimum length scale in either the solid phase or the void phase, but not both.
\end{abstract}

Keywords Topology optimization · Morphological filters · Length scale control $\cdot$ Sensitivity analysis

\section{Introduction}

In density based topology optimization (Bendsøe, 1989; Zhou and Rozvany, 1991), filters are commonly used in order to avoid checkerboard patterns (Díaz and Sigmund, 1995) and to ensure mesh independency (Jog and Haber, 1996).

\section{Schevenels}

Department of Architecture, Faculty of Engineering Science, KU Leuven, Kasteelpark Arenberg 1, 3001 Leuven, Belgium

E-mail: mattias.schevenels@kuleuven.be

O. Sigmund

Department of Mechanical Engineering, Solid Mechanics, Technical University of Denmark, Nils Koppel's Allé, Building 404, 2800, Lyngby, Denmark

E-mail: sigmund@mek.dtu.dk
Many filters are based on an averaging operation where the density of each element is replaced with the weighted average of the densities of the elements in its neighborhood (Bruns and Tortorelli, 2001; Bourdin, 2001). ${ }^{1}$ The averaging operation effectively removes small-scale features, but it also leads to gray transition zones between the solid (unit density, or black) and void (zero density, or white) phases. In order to remove these transition zones and to obtain crisp black-andwhite designs, the averaging operation is often followed by a Heaviside projection, which is an element-wise operation that maps intermediate densities to solid or void (Guest et al, 2004; Xu et al, 2010). In order to ensure that the optimization problem remains differentiable, a smoothed version of the Heaviside function is used for the projection. A projection threshold is defined to differentiate between solid and void: element densities smaller than the threshold value are projected to zero; element densities larger than the threshold value are projected to one. If the projection threshold itself is equal to zero, the resulting design has a minimum length scale in the solid phase. If the projection threshold is equal to one, the resulting design has a minimum length scale in the void phase. For intermediate values, neither in the solid phase nor in the void phase a minimum length scale is obtained.

As an alternative to Heaviside projection filters, Sigmund (2007) introduced density filters based on morphological operators used in image processing (Pratt, 1991; Bovik, 2009). The two basic operators are the dilation filter and the erosion filter. The former replaces the density of each element with the maximum density that occurs in its neighborhood, leading to a dilation of the solid phase; the latter uses the

1 In earlier publications, sensitivity filtering is performed instead of density filtering, which means that the averaging operation is applied to the sensitivities of the objective function and the constraints before they are passed to the optimizer to perform a design update (Sigmund, 1994, 1997; Sigmund and Petersson, 1998). 
minimum density, leading to an erosion of the solid phase. The dilation and erosion filter are often combined to form the close filter (dilation followed by erosion) and the open filter (erosion followed by dilation). The close filter removes all small features (and thus ensures a minimum length scale) in the void phase; the open filter does the same in the solid phase. In the 2007 paper, the close-open filter (opening followed by closing) and the open-close filter (closing followed by opening) were proposed in order to ensure a minimum length scale in both the solid and the void phase. However, it was concluded that these filters were not usable due to the immense computational cost of the sensitivity analysis. As a consequence, their performance in terms of length scale control could not be investigated in detail.

Later on, Sigmund (2009) introduced a robust formulation where the worst case of a dilated, an intermediate, and an eroded design is considered in the optimization. While this approach has originally been proposed in order to ensure robustness with respect to manufacturing errors, it also performs very well as a method to control the length scale in both the solid and the void phases (Wang et al, 2011). Very recently, Zhou et al. (2015) developed a similar method for length scale control by taking only the performance of the intermediate design into account in the optimization and by imposing additional constraints in order to ensure that the dilated, intermediate, and eroded design share the same topology. This approach leads to better performing (but less robust) designs than the original formulation.

While the problem of length scale control seems to be solved, we still believe it is worthwhile to reconsider the close-open and open-close filters, as they seem to provide a length scale control method that is easy to understand and that involves less parameters than the method proposed by Zhou et al. (2015). Moreover, we found that the sensitivity analysis for these filters can be performed much more efficiently than initially assumed, in a very straightforward manner, without the use of sophisticated techniques such as the method recently proposed by Wadbro and Hägg (2015).

This note is organized as follows. In section 2, the implementation of close-open and open-close filters and the (efficient) evaluation of the sensitivities are addressed. Section 3 discusses the application of the filters for the optimization of a compliant force inverter. Section 4 focuses more in detail on the sequential use of open and close filters with the intention of generating designs with a minimum length scale in both the solid and the void phase.

\section{Implementation}

This section focuses on the implementation of morphological filters for topology optimization and the calculation of the corresponding sensitivities. First, the dilation and erosion filters are addressed. Next, these filters are combined in order to form the close-open and open-close filters.

\subsection{Dilation filter}

In its discrete form, the dilation filter corresponds to a max operator: it replaces the density of each element with the maximum density occurring in its neighborhood. The discrete form does not allow for the use of a gradient based optimization scheme; it is therefore replaced with a similar continuous form by means of the Kreisselmeier-Steinhauser formulation (1983). The filtered density $\tilde{\rho}_{e}$ of element $e$ is thus obtained in terms of the unfiltered densities $\rho_{j}$ as:

$\tilde{\rho}_{e}=\log \left(\frac{\sum_{j} w_{e j} e^{\beta \rho_{j}}}{\sum_{j} w_{e j}}\right) / \beta$

where the kernel $w_{e j}$ determines the shape of the so-called structuring element of the filter; $w_{e j}$ equals one if the element $j$ is in the neighborhood of element $e$ and zero otherwise. The parameter $\beta$ controls the smoothness of the KreisselmeierSteinhauser formulation; for $\beta$ approaching zero, the filter corresponds to an averaging operation, for $\beta$ approaching infinity, it corresponds to the max operator.

In a gradient based optimization framework, we need an expression for the sensitivity $\frac{\partial f}{\partial \rho_{k}}$ of a (constraint or objective) function $f(\tilde{\boldsymbol{\rho}})$ with respect to the unfiltered density $\rho_{k}$ of each element $k$, as these unfiltered densities serve as design variables in the optimization problem. This sensitivity is obtained as follows:

$\frac{\partial f}{\partial \rho_{k}}=\sum_{e} \frac{\partial f}{\partial \tilde{\rho}_{e}} \frac{\partial \tilde{\rho}_{e}}{\partial \rho_{k}}$

where the derivative $\frac{\partial \tilde{\rho}_{e}}{\partial \rho_{k}}$ is given by:

$\frac{\partial \tilde{\rho}_{e}}{\partial \rho_{k}}=\frac{w_{e k} e^{\beta \rho_{k}}}{\sum_{j} w_{e j} e^{\beta \rho_{j}}}$

The expressions on the right hand side of equations (1) and (3) both consist of a linear averaging operation preceded and succeeded a non-linear element-wise operation. The averaging operation represents the highest computational cost, but it can be efficiently implemented as a convolution (Andreassen et al, 2011) or by means of a specialized technique where averages over overlapping parts of different neighborhoods are computed only once (Wadbro and Hägg, 2015). If the filter kernel $w_{e j}$ would correspond to the Green's function of a PDE (which is not the case, as the kernel considered here has a compact basis), the operation could also be efficiently performed by means of a PDE based filtering technique (Lazarov and Sigmund, 2011). 


\subsection{Erosion filter}

The erosion filter corresponds to the min operator, which is again replaced with a similar continuous form by means of the Kreisselmeier-Steinhauser formulation. The resulting densities are denoted as $\bar{\rho}_{e}$; they are obtained as:

$\bar{\rho}_{e}=1-\log \left(\frac{\sum_{j} w_{e j} e^{\beta\left(1-\rho_{j}\right)}}{\sum_{j} w_{e j}}\right) / \beta$

The sensitivity $\frac{\partial f}{\partial \rho_{k}}$ of a function $f(\overline{\boldsymbol{\rho}})$ with respect to the $k$-th design variable $\rho_{k}$ is given by:

$\frac{\partial f}{\partial \rho_{k}}=\sum_{e} \frac{\partial f}{\partial \bar{\rho}_{e}} \frac{\partial \bar{\rho}_{e}}{\partial \rho_{k}}$

where the derivative $\frac{\partial \bar{\rho}_{e}}{\partial \rho_{k}}$ is obtained as:

$\frac{\partial \bar{\rho}_{e}}{\partial \rho_{k}}=\frac{w_{e k} e^{\beta\left(1-\rho_{k}\right)}}{\sum_{j} w_{e j} e^{\beta\left(1-\rho_{j}\right)}}$

The implementation strategy proposed for the dilation filter is also applicable to the erosion filter.

\subsection{Close-open and open-close filters}

The erosion and dilation filters may be applied sequentially in order to form close, open, close-open, and open-close filters. The close filter is defined as a dilation followed by an erosion, the open filter as an erosion followed by a dilation, the close-open filter as opening followed by closing, and the open-close filter as closing followed by opening. ${ }^{2}$

As an example, the open-close filter is discussed. The physical densities obtained with the open-close filter are denoted by $\tilde{\overline{\tilde{\rho}}}_{e}$. They are computed in four steps:

$\tilde{\rho}_{e}=\log \left(\frac{\sum_{j} w_{e j} e^{\beta \rho_{j}}}{\sum_{j} w_{e j}}\right) / \beta$

$\overline{\tilde{\rho}}_{e}=1-\log \left(\frac{\sum_{j} w_{e j} e^{\beta\left(1-\tilde{\rho}_{j}\right)}}{\sum_{j} w_{e j}}\right) / \beta$

$\overline{\tilde{\tilde{\rho}}}_{e}=1-\log \left(\frac{\sum_{j} w_{e j} e^{\beta\left(1-\tilde{\tilde{\rho}}_{j}\right)}}{\sum_{j} w_{e j}}\right) / \beta$

$\tilde{\overline{\tilde{\rho}}}_{e}=\log \left(\frac{\sum_{j} w_{e j} e^{\beta \overline{\tilde{\rho}}_{j}}}{\sum_{j} w_{e j}}\right) / \beta$

2 The definitions of the close-open and open-close filters might seem counterintuitive, but these definitions are used in the literature on image processing (Bovik, 2009). In the original paper (Sigmund, 2007), the definitions of the close-open and open-close filters are swapped in the text, but the results are denoted correctly.
The sensitivity $\frac{\partial f}{\partial \rho_{k}}$ of a function $f(\tilde{\overline{\tilde{\boldsymbol{\rho}}}})$ with respect to the $k$-th design variable $\rho_{k}$ is obtained by applying the chain rule four times:

$$
\begin{aligned}
& \frac{\partial f}{\partial \overline{\tilde{\tilde{\rho}}}_{k}}=\sum_{e} \frac{\partial f}{\partial \tilde{\overline{\tilde{\rho}}}_{e}} \frac{\partial \tilde{\overline{\tilde{\rho}}}_{e}}{\partial \overline{\tilde{\tilde{\rho}}}_{k}} \\
& \frac{\partial f}{\partial \overline{\tilde{\rho}}_{k}}=\sum_{e} \frac{\partial f}{\partial \overline{\tilde{\tilde{\rho}}}_{e}} \frac{\partial \overline{\tilde{\tilde{\rho}}}_{e}}{\partial \overline{\tilde{\rho}}_{k}} \\
& \frac{\partial f}{\partial \tilde{\rho}_{k}}=\sum_{e} \frac{\partial f}{\partial \overline{\tilde{\rho}}_{e}} \frac{\partial \overline{\tilde{\rho}}_{e}}{\partial \tilde{\rho}_{k}} \\
& \frac{\partial f}{\partial \rho_{k}}=\sum_{e} \frac{\partial f}{\partial \tilde{\rho}_{e}} \frac{\partial \tilde{\rho}_{e}}{\partial \rho_{k}}
\end{aligned}
$$

or:

$$
\frac{\partial f}{\partial \rho_{k}}=\sum_{e} \sum_{p} \sum_{q} \sum_{r} \frac{\partial f}{\partial \tilde{\overline{\tilde{\rho}}}_{r}} \frac{\partial \tilde{\tilde{\tilde{\rho}}}_{r}}{\partial \overline{\tilde{\tilde{\rho}}}_{q}} \frac{\partial \overline{\tilde{\tilde{\rho}}}_{q}}{\partial \overline{\tilde{\rho}}_{p}} \frac{\partial \overline{\tilde{\rho}}_{p}}{\partial \tilde{\rho}_{e}} \frac{\partial \tilde{\rho}_{e}}{\partial \rho_{k}}
$$

In the original paper (Sigmund, 2007), the evaluation of equation (15) is considered to be computationally extremely expensive due to the nested nature of the summation operators. However, the high computational cost is only due to the fact that the partial derivatives $\frac{\partial \tilde{\overline{\tilde{\rho}}}_{r}}{\partial \overline{\bar{\rho}}_{q}} \frac{\partial \overline{\tilde{\tilde{\rho}}}_{q}}{\partial \tilde{\tilde{\rho}}_{p}}, \frac{\partial \overline{\tilde{\rho}}_{p}}{\partial \tilde{\rho}_{e}}$, and $\frac{\partial \tilde{\rho}_{e}}{\partial \rho_{k}}$ and their products are recomputed in every summation step. It is much more efficient to compute the sensitivities $\frac{\partial f}{\partial \rho_{k}}$ in four steps, corresponding to equations (11-14), so avoiding to perform the same computations over and over again. The number of summation operations then reduces from $n s^{4}$ as reported in the original paper to $4 n s$, where $n$ is the number of finite elements in the design domain and $s$ is the size of the structuring element (in terms of finite elements) used for the filtering operation.

While the formulation considered in this note is based on morphological operators, it is also possible to define closeopen and open-close filters based on sequential Heaviside projections. In that case, the same strategy can be followed to perform the sensitivity analysis in an efficient way.

\section{Test example}

\subsection{Problem formulation}

This section addresses the optimization of a compliant force inverter. The design domain and boundary conditions are shown in figure 1 . The objective is to maximize the displacement $u_{\text {out }}$ in the output degree-of-freedom for a unit input force $f_{\text {in }}$. The amount of material is limited to $25 \%$ of the design domain. The design domain is discretized using $n=300 \times 150=45000$ square finite elements with unit dimensions. The Young's modulus is $E_{0}=1$ for the solid 


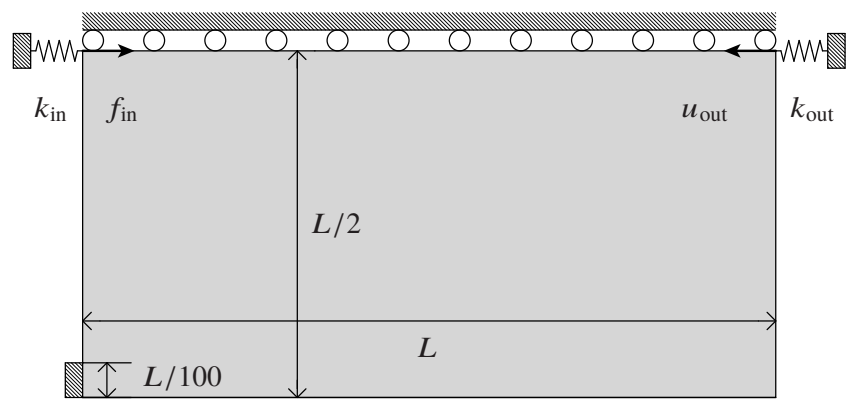

Fig. 1 Design domain and boundary conditions for the force inverter.

phase and $E_{\min }=10^{-9}$ for the void phase, and Poisson's ratio is $v=0.3$. The spring stiffnesses of the input and output springs are $k_{\text {in }}=0.4$ and $k_{\text {out }}=0.0004$, respectively.

The modified SIMP interpolation scheme proposed by Sigmund (2007) with a penalization power $p=3$ is used to relate the Young's modulus of an element to its physical density. As in the original paper, the value of $p$ remains fixed throughout the optimization. The physical densities are computed using (1) a close-open filter and (2) an open-close filter. In both cases, a circular structuring element with a radius $R=6.25$ is used. The size of this structuring element is $s=121$. This means that the number of summation operations in the sensitivity analysis would be equal to $n s^{4}=9.6 \times 10^{12}$ according to the originally proposed procedure, while it is equal to $4 n s=21.8 \times 10^{6}$ if the procedure proposed in this note is followed.

A conservative continuation scheme is followed for the filter's smoothness parameter $\beta$ in order to ensure smooth convergence of the optimization algorithm: in the first iteration, a value $\beta=0.2$ is used, and in every subsequent iteration, the value of $\beta$ is multiplied with a factor 1.01, until it becomes larger than 200. This occurs after 696 iterations. From then on, the value of $\beta$ is kept constant. Initially, the entire design domain is assumed to be solid. The optimization is performed using the Method of Moving Asymptotes (MMA) (Svanberg, 1987), and the algorithm is terminated after 1000 iterations.

\subsection{Results}

Figure 2 shows the convergence history of the objective function during the optimization of the inverter using a closeopen filter and an open-close filter. It can be observed that the objective function reaches an almost constant level after about 100 iterations. At this point, the final design has been obtained, except that there are still gray transition zones between the solid and the void phase. These transition zones start to disappear as soon as the smoothness parameter $\beta$ becomes sufficiently high, resulting in a further increase of the objective function. Once the value of $\beta$ is fixed, the objective function remains almost constant.

In terms of objective value and measure of nondiscreteness (Sigmund, 2007), both the close-open filter and the open-close filter yield good results: in the case of the close-open filter, the final value of the objective function is $u_{\text {out }}=8.28$, and the measure of non-discreteness is $M_{\text {nd }}=2.36 \%$. In the case of the open-close filter, the final value of the objective function is $u_{\text {out }}=8.01$, and the measure of non-discreteness is $M_{\mathrm{nd}}=2.56 \%$. In both cases, the material volume constraint is exactly satisfied.

The intention of implementing the close-open and openclose filters was to obtain a design with a minimum length scale in both the solid and the void phase; that is, a design that can be produced both by material deposition and by machining, using a tool with the shape of the structuring element adopted in the filtering procedure. In order to assess whether this goal has been achieved, figure 3 shows the optimized force inverter using the two types of filters. The shape of the structuring element is also shown.

The close-open filter leads to a design with a minimum length scale in the void phase, but not in the solid phase: very thin connections occur that will mimic the effect of structural hinges. Conversely, the open-close filter leads to a design with a minimum length scale in the solid phase, but not in the void phase: the design consists of several parts, connected by (unproducible) single-node hinges.

It must be concluded that the close-open and open-close filters allow for length scale control in either the solid phase or the void phase, but not both, which may seem counterintuitive. In the next section, the filters are studied in more detail in order to come up with an explanation for this conclusion.

\section{Length scale}

The test example addressed in the previous section demonstrates that the use of close-open and open-close filters does not (always) lead to a design with a minimum length scale in both the solid and the void phase. The question then arises whether this is inherent to the close-open and open-close filters or whether it is due to the fact that we use smooth versions of these filters (in which case changing the continuation scheme or switching to Heaviside based close-open and open-close filters could possibly offer a solution). This section addresses the length scale problem in more detail.

An unsmoothed version of the close-open as well as the open-close filter is applied to the black-and-white test pattern shown in figures 4(1a) and 4(2a). The domain is discretized using $1000 \times 1000$ square elements of unit size, and a circular structuring element with a radius $R=40$ is used for both filters. The results are shown in figure 4.

Figures $4(1 e)$ and 4(2c) show an outcome of the close filter. These figures seem to confirm that the close filter elim- 


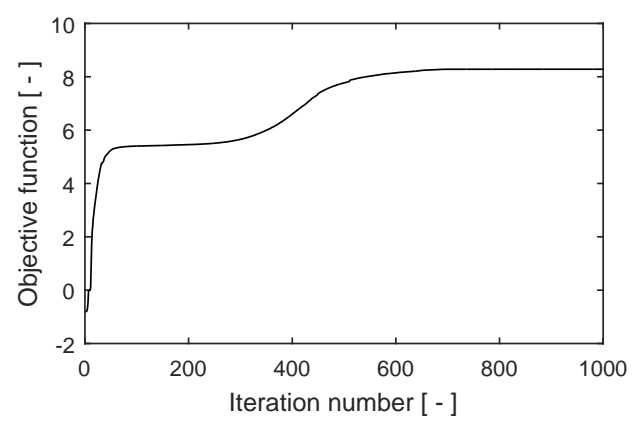

(a) Close-open filter

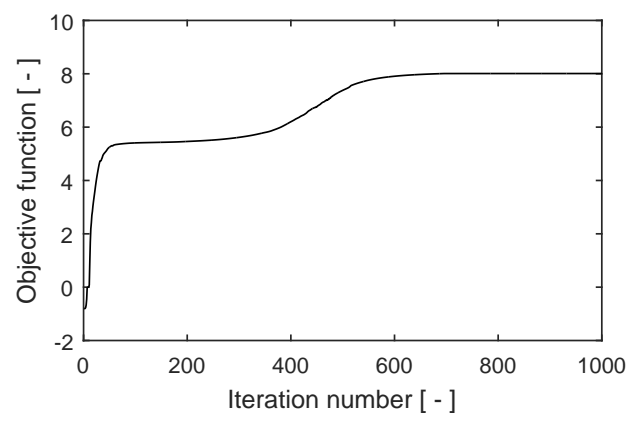

(b) Open-close filter

Fig. 2 Convergence history of the objective function for the inverter test example obtained using (a) the close-open filter and (b) the open-close filter.

Structuring element
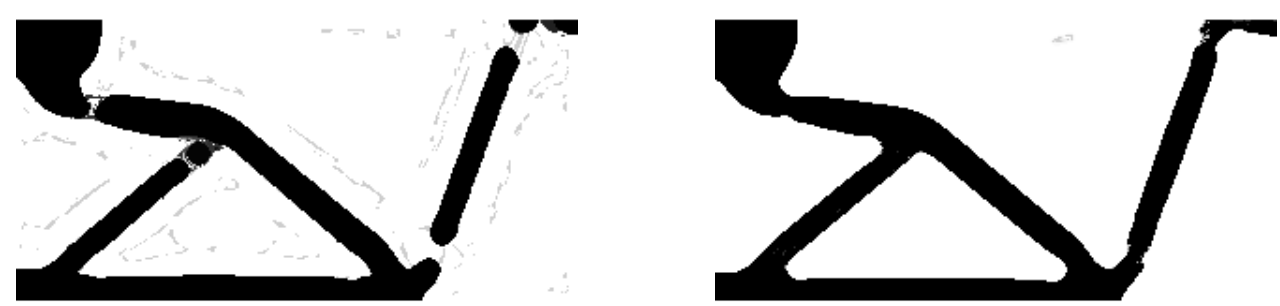

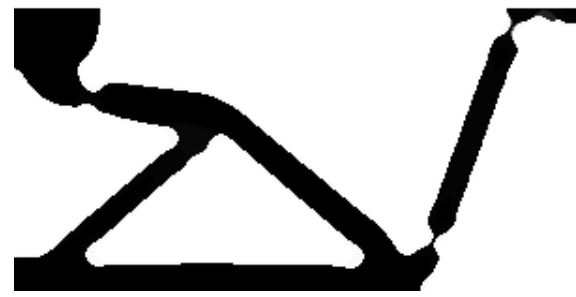

(a) Close-open filter

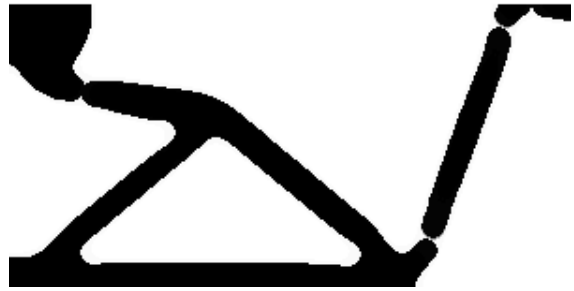

(b) Open-close filter

Fig. 3 Results for the inverter test example obtained using (a) the close-open filter and (b) the open-close filter. For both filters, two images are shown. The upper image shows the (non-physical) design variable field; the lower image shows the (physical) filtered density field.

inates small holes and thus ensures a minimum length scale in the void phase (Sigmund, 2007). Likewise, figures 4(1c) and 4(2e) show an outcome of the open filter; they confirm that the open filter eliminates small solid features and leads to a design with a minimum length scale in the solid phase. However, the effect on the length scale is not preserved by subsequent filters. Figure 4(1e) clearly demonstrates that the close filter ruins the effect of the open filter: the length scale in the solid phase is completely lost. It can therefore be concluded that the use of an open-close filter does not lead to a design with a minimum length scale in both phases. By inverting the input pattern, one can draw the same conclusion for the close-open filter.

While the example pattern used here might be considered pathological, it helps explaining the small features that occur in the force inverter considered in the previous section, and it demonstrates that the use of a close-open filter or an open-close filter does not guarantee the existence of a length scale in both solid and void phases. As this is true for the unsmoothed version of the filters and for a completely blackand-white input pattern, it can be concluded that changing the continuation scheme or switching to Heaviside based close-open or open-close filters would offer no relief.

\section{Conclusion}

This note reconsiders the morphological close-open and open-close filters for topology optimization introduced in an earlier paper (Sigmund, 2007). The implementation of the filters (and, especially, the computation of the sensitivities) is addressed, and the performance of the filters in terms of length scale control is investigated.

It is shown that the sensitivities of the close-open and open-close filters can be computed much more efficiently 
(1)

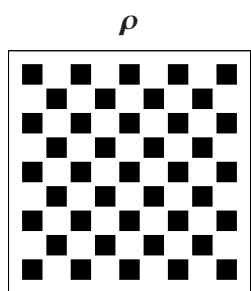

$\rho$

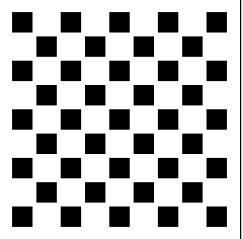

(a)

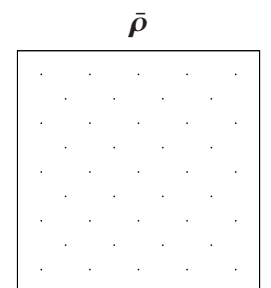

$\tilde{\rho}$

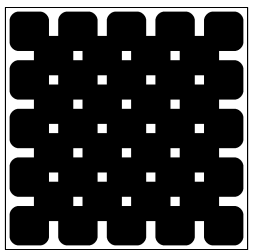

(b)

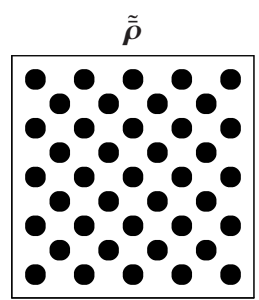

$\overline{\tilde{\rho}}$

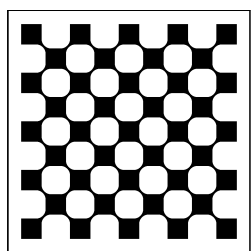

(c)
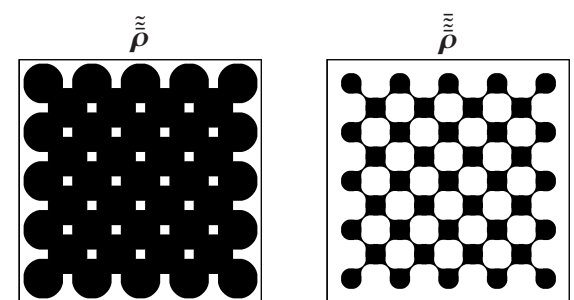

$\overline{\tilde{\tilde{\rho}}}$

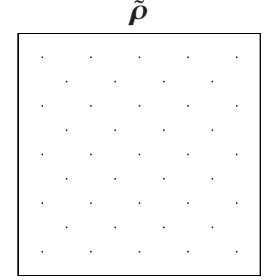

(d)

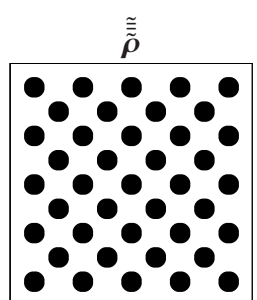

(e)

Fig. 4 (1) Application of an unsmoothed close-open filter to a test pattern; from left to right: design variables $\boldsymbol{\rho}$, intermediate variables $\overline{\boldsymbol{\rho}}$ after first erosion, intermediate variables $\tilde{\tilde{\boldsymbol{\rho}}}$ after first dilation, intermediate variables $\tilde{\tilde{\boldsymbol{\rho}}}$ after second dilation, and physical densities $\overline{\tilde{\tilde{\boldsymbol{\rho}}}}$ after second erosion; and (2) application of an unsmoothed open-close filter to a test pattern; from left to right: design variables $\boldsymbol{\rho}$, intermediate variables $\tilde{\boldsymbol{\rho}}$ after first dilation, intermediate variables $\overline{\tilde{\boldsymbol{\rho}}}$ after first erosion, intermediate variables $\overline{\overline{\tilde{\boldsymbol{\rho}}}}$ after second erosion, and physical densities $\tilde{\overline{\tilde{\boldsymbol{\rho}}}}$ after second dilation.

than initally assumed by evaluating the chain rule in a stepby-step approach.

Unfortunately, the open-close and close-open filters do not impose a length scale on both the solid and the void fraction. Each close or open operation ruins the effect of the preceding filters. As a consequence, the close-open filter (where the last step is a close operation) only guarantees a minimum length scale in the void phase, and the open-close filter (where the last step is an open operation) only guarantees a minimum length scale in the solid phase. If length scale control in both phases is required, the robust approach (Wang et al, 2011) and the method recently proposed by Zhou et al. (2015) have proven to be more effective.

\section{References}

Andreassen E, Clausen A, Schevenels M, Lazarov B, Sigmund O (2011) Efficient topology optimization in MATLAB using 88 lines of code. Structural and Multidisciplinary Optimization 43:1-16, URL http://dx.doi.org/10.1007/s00158-010-0594-7

Bendsøe M (1989) Optimal shape design as a material distribution problem. Structural and Multidisciplinary Optimization 1(4):193-202

Bourdin B (2001) Filters in topology optimization. International Journal for Numerical Methods in Engineering 50(9):2143-2158

Bovik A (2009) The essential guide to image processing. Academic Press

Bruns T, Tortorelli D (2001) Topology optimization of nonlinear elastic structures and compliant mechanisms. Com- puter Methods in Applied Mechanics and Engineering 190(26-27):3443-3459

Díaz A, Sigmund O (1995) Checkerboard patterns in layout optimization. Structural Optimization 10(1):40-45

Guest J, Prevost J, Belytschko T (2004) Achieving minimum length scale in topology optimization using nodal design variables and projection functions. International Journal for Numerical Methods in Engineering 61(2):238-254

Jog C, Haber R (1996) Stability of finite element models for distributed-parameter optimization and topology design. Computer Methods in Applied Mechanics and Engineering 130(3-4):203-226

Kreisselmeier G, Steinhauser R (1983) Application of vector performance optimization to a robust-control loop design for a fighter aircraft. International Journal of Control 37(2):251-284

Lazarov BS, Sigmund O (2011) Filters in topology optimization based on helmholtz-type differential equations. International Journal for Numerical Methods in Engineering 86(6):765-781

Pratt W (1991) Digital image processing. Wiley

Sigmund O (1994) Design of material structures using topology optimization. PhD thesis, Department of Solid Mechanics, Technical University of Denmark

Sigmund O (1997) On the design of compliant mechanisms using topology optimization. Mechanics of Structures and Machines 25(4):493-524

Sigmund O (2007) Morphology-based black and white filters for topology optimization. Structural and Multidisciplinary Optimization 33(4-5):401-424

Sigmund O (2009) Manufacturing tolerant topology optimization. Acta Mechanica Sinica 25(2):227-239 
Sigmund O, Petersson J (1998) Numerical instabilities in topology optimization: A survey on procedures dealing with checkerboards, mesh-dependencies and local minima. Structural Optimization 16(1):68-75

Svanberg K (1987) The method of moving asymptotes a new method for structural optimization. International Journal for Numerical Methods in Engineering 24(2):359373

Wadbro E, Hägg L (2015) On quasi-arithmetic mean based filters and their fast evaluation for large-scale topology optimization. Structural and Multidisciplinary Optimization Published online

Wang F, Lazarov B, Sigmund O (2011) On projection methods, convergence and robust formulations in topology optimization. Structural and Multidisciplinary Optimization 43(6):767-784

Xu S, Cai Y, Cheng G (2010) Volume preserving nonlinear density filter based on Heaviside functions. Structural and Multidisciplinary Optimization 41(4):495-505

Zhou M, Rozvany G (1991) The COC algorithm, part II: Topological, geometrical and generalized shape optimization. Computer Methods in Applied Mechanics and Engineering 89(1-3):309-336

Zhou M, Lazarov B, Wang F, Sigmund O (2015) Minimum length scale in topology optimization by geometric constraints. Computer Methods in Applied Mechanics and Engineering 293:266-282 